\title{
Matrix and cell phenotype differences in Dupuytren's disease
}

\author{
Marike M. van Beuge ${ }^{1 *}$, Evert-Jan P. M. ten Dam ${ }^{1,2}$, Paul M. N. Werker ${ }^{2}$ and Ruud A. Bank ${ }^{1}$
}

\begin{abstract}
Background: Dupuytren's disease is a fibroproliferative disease of the hand and fingers, which usually manifests as two different phenotypes within the same patient. The disease first causes a nodule in the palm of the hand, while later, a cord develops, causing contracture of the fingers.

Results: We set out to characterize the two phenotypes by comparing matched cord and nodule tissue from ten Dupuytren's patients. We found that nodule tissue contained more proliferating cells, CD68-positive macrophages and a-smooth muscle actin (a-SMA)-positive myofibroblastic cells. qPCR analysis showed an increased expression of COL1A1, COL1A2, COL5A1, and COL6A1 in nodule tissue compared to cord tissue. Immunohistochemistry showed less deposition of collagen type I in nodules, although they contained more fibronectin, collagen type $\mathrm{V}$, and procollagen 1. Lower collagen levels in nodule were confirmed by HPLC measurements of the Hyp/Pro ratio. PCOLCE2, an activator of BMP1, the main enzyme cleaving the C-terminal pro-peptide from procollagen, was also reduced in nodule. Cord tissue not only contained more collagen I, but also higher levels of hydroxylysylpyridinoline and lysylpyridinoline residues per triple helix, indicating more crosslinks.

Conclusions: Our results clearly show that in Dupuytren's disease, the nodule is the active disease unit, although it does not have the highest collagen protein levels. The difference in collagen type I deposition compared to mRNA levels and procollagen 1 levels may be connected to a decrease in procollagen processing.
\end{abstract}

Keywords: Dupuytren's disease, Fibroblast, Collagen biosynthesis, PCOLCE2

\section{Background}

Any fibrotic process is an interplay between cells and matrix, with the matrix influencing cell proliferation, adhesion, and migration, and the cells influencing matrix composition and crosslinking [1]. This process is the ultimate consequence of a range of insults and can occur in virtually all organs of the body, with many differences and similarities between the organs. These differences may depend on the regenerative capacity of the organ or its capacity to compensate for the loss of function due to the build-up of extracellular matrix. All fibrotic diseases are characterized by the presence of myofibroblasts, a cell type containing abundant actin fibers. These cells are capable of producing and depositing large amounts of excess

\footnotetext{
* Correspondence: m.m.van.beuge@umcg.nl

'Department of Pathology \& Medical Biology, University Medical Center Groningen, University of Groningen, Hanzeplein 1, 9713 GZ Groningen, The Netherlands

Full list of author information is available at the end of the article
}

extracellular matrix, and their presence is therefore considered a main cause of fibrotic disease [2].

Dupuytren's disease is a very common (prevalence 0.6 to $31.6 \%$ [3]) fibroproliferative disease of the hand and fingers, which generally starts with the formation of a nodule in the palm of the hand, and progresses with the formation of a cord towards the fingers, which causes eventual contraction and the inability to extend the fingers. These two phenotypes commonly occur together in the same patient [4].

Previous studies have addressed the differences between cord and nodule and found that there are differences in contractility of isolated fibroblasts, with cells isolated from nodule being more contractile in vitro [5]. Furthermore, these cells express more $\alpha$-smooth muscle actin [6]. A large majority of these studies however was performed on isolated, subcultured fibroblasts, which may grossly distort the phenotype of the cells [7]. Studies on complete tissue have shown that cord and nodule have a different ratio of expression of matrix metalloproteinases (MMPs) 
and tissue inhibitors of matrix metalloproteinases (TIMPs) $[8,9]$. Older studies have reported differences in the type of extracellular matrix that is deposited in nodules or cords, with the former containing more fibronectin, laminin, collagen IV, and tenascin $\mathrm{C}$, particularly in proliferative areas [10-12].

In a different classification, devised first by Luck [13], Dupuytren's tissue has also been reported to contain several zones, which can be distinguished histologically as the proliferative, involutional, and residual zone. The proliferative zone is characterized by the presence of a large number of (myo)fibroblasts, the involutional zone contains large amounts of collagens and myofibroblasts, and the residual zone is relatively poor in cells.

Dupuytren's disease displays the relatively rare phenomenon of two phenotypes (e.g., cord and nodule) at the same time in the same patient, with much discussion still going on in the field as to the origins of these tissues [14]. We investigated the differences between these phenotypes at a molecular level. Specifically, we wanted to address the question of whether the difference is a difference of the cell types present and/or a difference in the composition and amount of extracellular matrix. Differences in extracellular matrix (ECM) constituents and density between cord and nodule may account for pathogenesis of the two phenotypes and may influence current and future treatment options.

\section{Results}

\section{Proliferation and cell types in cord versus nodule}

In order to determine whether the differences between cord and nodule are determined by the number, type, and activity of the cells present, we performed several immunohistochemical stainings. Staining for Ki-67, which is expressed in the nuclei of proliferating cells, showed that the number of proliferating cells was significantly higher in nodule tissue compared to cord tissue of the same patients (Fig. 1a). Furthermore, nodular tissue contained more $\alpha$-smooth muscle actin ( $\alpha$-SMA), a marker for myofibroblasts (Fig. 1b), and more CD68-positive cells (Fig. 1c), which denotes mainly macrophages. We found no significant difference in the area of CD31-positive cells, indicating that there is no difference in the number of blood vessels between cord and nodule (Fig. 1d).

\section{Extracellular matrix production}

To study the differences in the production of extracellular matrix between cord and nodule of Dupuytren's patients, we first performed a gene expression analysis on extracellular matrix components and biosynthesis molecules. In total, we examined the expression of 44 genes; full results of this analysis can be found in Table 1; notable findings are discussed below.
We found a significantly higher expression of COL1A1, COL1A2, COL5A1, and COL6A1 messenger RNA (mRNA) in nodule tissue (Fig. 2a). There were no significant differences in gene expression of COL3A1 and COL4A1. In addition, five non-collagenous extracellular matrix molecules were studied; of these, there was only a significant difference in the expression of FN1, which was higher in nodule (Fig. 2b). There were no differences in the expression of BGN, DCN, ELN, and FMOD.

\section{Extracellular matrix deposition}

To study whether the differences we found in mRNA levels of ECM molecules also resulted in differences in the composition of the deposited ECM, we performed immunohistochemical analyses. We found significantly more fibronectin deposition in nodules compared to cords (Fig. 3a), whereas there was no difference in levels of tenascin $C$, which was earlier reported to be increased in nodules as well (Fig. 3b, [10]). However, we found more elastin present in nodules compared to cords (Fig. 3c).

Analysis of collagen deposition showed that, in contrast to the mRNA data, collagen type I deposition in cord was significantly higher than in nodule (Fig. 4a), whereas there was no difference in the deposition of collagen type III (Fig. 4b), and a significant increase in collagen type $\mathrm{V}$ in nodule compared to cord (Fig. 4c). Contrary to collagen, a higher expression of procollagen type I is seen in nodule than in cord (Fig. 4d), which does correspond to the mRNA results. To verify our immunohistochemical data on collagen, we quantified the total amount of collagen in the tissues by measuring the Hyp/Pro ratio using HPLC. Increased collagen levels result in increased Hyp levels compared to Pro, giving rise to higher Hyp/Pro ratios [15]. Since collagen type I is the major collagen type in cords and nodules, one would expect (based on the collagen type I immunohistochemistry staining) in cord samples a higher Hyp/Pro ratio compared to nodule samples. This was indeed the case (Fig. 4e).

\section{Collagen I processing}

In view of the discrepancy between the mRNA levels of COL1A1 and COL1A2, which were higher in nodule and the observed deposition of collagen I which was higher in cord, we decided to further investigate this phenomenon. Analysis of mRNA expression of enzymes involved in collagen synthesis revealed an upregulation of LEPREL2, which has prolyl-3-hydroxylation activity, and $\mathrm{P} 4 \mathrm{HB}$, which catalyzes the formation of 4-hydroxyproline, both in nodule (Fig. 5a). Therefore, collagen synthesis may be slightly higher in nodule, depending on the relative activities of the different 3- and 4-hydroxylation enzymes. We then investigated procollagen processing and found that the expression of ADAMTS14 was significantly higher in nodule but very variable among patients. Levels 


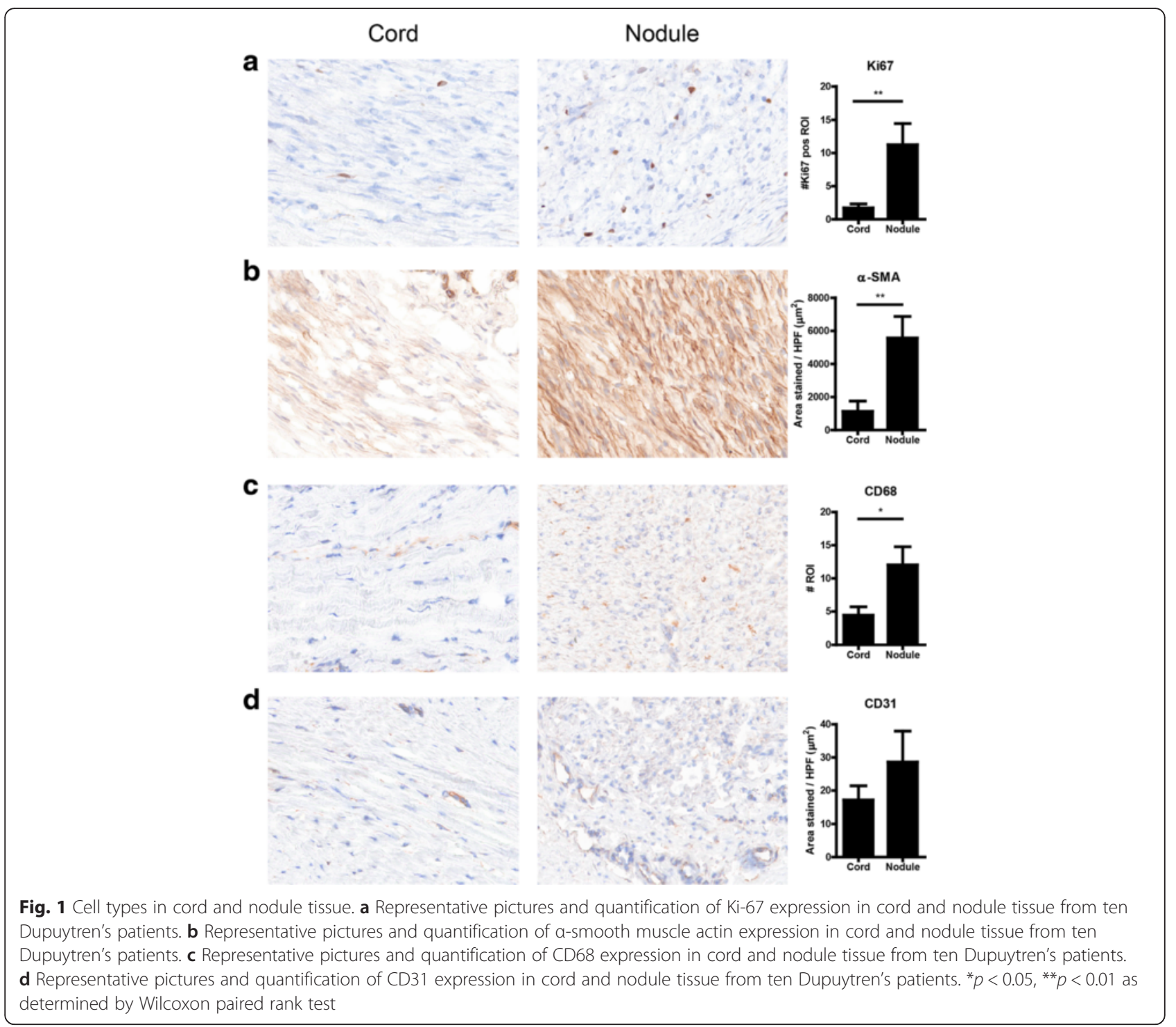

of ADAMTS2 and ADAMTS3 were unchanged (Fig. 5b). There was no difference in the expression of BMP1, either at mRNA or protein level (Fig. 5c, d). However, we found a consistently lower mRNA expression of PCOLCE2, which activates BMP1, in nodules compared to cord (Fig. 5c), which was also confirmed at protein level by immunohistochemistry (Fig. 5e).

\section{Crosslinked collagen}

The composition of the deposited collagen was examined by HPLC analysis of crosslinks, corrected for the amount of collagen present, expressed as number of hydroxylysylpyridinoline (HP) and lysylpyridinoline (LP) residues per triple helix. We found a significant increase of both types of crosslinks in cord tissue compared to nodule tissue (Fig. 6). In contradiction, of the main enzymes responsible for collagen crosslinking, we only found a significant difference in the expression of LOX, which was higher in nodule, whereas the expression of LOXL1-4 and PLOD1-3 was unchanged (Table 1).

\section{Discussion}

In this study, we showed that there are considerable differences between cord and nodule at mRNA and protein level, both in cell types present as well as in the extracellular matrix composition. These differences are probably interdependent and together are responsible for the disease phenotype as it develops in patients. To our knowledge, this is the first study comparing cell types, matrix deposition, and the collagen biosynthesis pathway in cord and nodule tissue concurrently.

In a previous study [13], a proliferative stage was proposed to exist in Dupuytren's disease, mainly in nodules, which coincides with our findings of higher Ki-67 
Table 1 Median expression levels and fold change of all genes analyzed in Dupuytren's nodule and matching cord tissue. $p$ value determined by Wilcoxon paired rank test

\begin{tabular}{|c|c|c|c|c|c|}
\hline Gene symbol & $N$ & Mean cord & Mean nodule & $\begin{array}{l}\text { Fold change } \\
\text { nodule/cord }\end{array}$ & $p$ value \\
\hline ADAMTS14 & 8 & 0.0041 & 0.0124 & 2.988 & 0.0234 \\
\hline ADAMTS2 & 8 & 0.2375 & 0.2983 & 1.256 & n.s. \\
\hline ADAMTS3 & 6 & 0.0025 & 0.0032 & 1.321 & n.s. \\
\hline BGN & 8 & 1.2588 & 1.5649 & 1.243 & n.s. \\
\hline BMP1 & 8 & 0.0424 & 0.0556 & 1.312 & n.s. \\
\hline COL1A1 & 8 & 4.2939 & 10.7051 & 2.493 & 0.0078 \\
\hline COL1A2 & 8 & 2.6263 & 4.7832 & 1.821 & 0.0078 \\
\hline COL3A1 & 8 & 3.7696 & 7.8217 & 2.075 & n.s. \\
\hline COL4A1 & 6 & 0.1868 & 0.1972 & 1.152 & n.s. \\
\hline COL5A1 & 8 & 0.3198 & 0.6450 & 2.017 & 0.0156 \\
\hline COL6A1 & 8 & 2.5967 & 4.1680 & 1.605 & 0.0234 \\
\hline COLGALT1 & 7 & 0.0386 & 0.0415 & 1.098 & n.s. \\
\hline CTSK & 7 & 0.1254 & 0.1927 & 1.742 & 0.0313 \\
\hline DCN & 8 & 0.7646 & 0.6807 & 0.890 & n.s. \\
\hline DDR1 & 6 & 0.0082 & 0.0068 & 0.768 & n.s. \\
\hline DDR2 & 8 & 0.0540 & 0.0421 & 0.780 & 0.0391 \\
\hline ELN & 8 & 0.0959 & 0.1054 & 1.099 & n.s. \\
\hline FKBP10 & 8 & 0.1019 & 0.1440 & 1.414 & 0.0234 \\
\hline FMOD & 8 & 0.1868 & 0.1334 & 0.714 & n.s. \\
\hline FN1 & 8 & 1.9316 & 3.4849 & 1.804 & 0.0078 \\
\hline LEPRE1 & 8 & 0.0140 & 0.0182 & 1.304 & n.s. \\
\hline LEPREL1 & 8 & 0.0023 & 0.0023 & 1.019 & n.s. \\
\hline LEPREL2 & 8 & 0.0154 & 0.0271 & 1.759 & 0.0156 \\
\hline LOX & 7 & 0.0425 & 0.0603 & 1.597 & 0.0156 \\
\hline LOXL1 & 8 & 0.0400 & 0.0555 & 1.388 & n.s. \\
\hline LOXL2 & 8 & 0.1436 & 0.2461 & 1.714 & n.s. \\
\hline LOXL3 & 7 & 0.0067 & 0.0057 & 0.782 & n.s. \\
\hline LOXL4 & 8 & 0.0015 & 0.0009 & 0.574 & n.s. \\
\hline MMP1 & 4 & 0.0004 & 0.0003 & 0.839 & n.s. \\
\hline MMP13 & 6 & 0.0015 & 0.0023 & 1.302 & n.s. \\
\hline MMP14 & 8 & 0.3076 & 0.6230 & 2.026 & n.s. \\
\hline MRC2 & 7 & 0.1996 & 0.2784 & 1.378 & 0.0313 \\
\hline P4HA1 & 6 & 0.0094 & 0.0114 & 1.217 & n.s. \\
\hline P4HA2 & 5 & 0.0009 & 0.0012 & 1.363 & n.s. \\
\hline P4HA3 & 8 & 0.0171 & 0.0338 & 1.980 & n.s. \\
\hline P4HB & 8 & 0.2390 & 0.3264 & 1.366 & 0.0234 \\
\hline PCOLCE & 7 & 0.4790 & 0.5545 & 1.231 & n.s. \\
\hline PCOLCE2 & 7 & 0.0326 & 0.0107 & 0.224 & 0.0313 \\
\hline PLOD1 & 8 & 0.0730 & 0.0985 & 1.350 & n.s. \\
\hline PLOD2 & 8 & 0.0248 & 0.0380 & 1.533 & n.s. \\
\hline PLOD3 & 7 & 0.0423 & 0.0419 & 0.964 & n.s. \\
\hline
\end{tabular}

Table 1 Median expression levels and fold change of all genes analyzed in Dupuytren's nodule and matching cord tissue. $p$ value determined by Wilcoxon paired rank test (Continued)

\begin{tabular}{llllll}
\hline SERPINH1 & 8 & 0.1895 & 0.2439 & 1.287 & n.s. \\
SLC39A13 & 6 & 0.0538 & 0.0505 & 1.022 & n.s. \\
TIMP1 & 8 & 0.1956 & 0.1915 & 0.979 & n.s. \\
\hline
\end{tabular}

expression in nodule. In an extension on this system, Lam et al. also found a correlation between collagen III expression and the stages proposed by Luck, with a higher proportion of collagen III in the proliferative stage [16]. In contrast, we did not find an increase in collagen III expression in nodule, which may be due to differences in the method of determination, i.e., immunohistochemistry in our study and a histochemical staining (Herovici's staining) used by Lam et al. Additionally, differences may be caused by the heterogeneity of cord tissue, which we noticed especially in our immunohistochemical studies, where sometimes we found small nodule-like structures within the cords, as has also been reported by others [17]. These were however not large enough to obscure the differences between the two types of tissue, although they are undoubtedly partly responsible for the variation seen between the samples.

In accordance with previous papers examining tissue from Dupuytren's patients, we found a significant amount of CD68-positive macrophages in nodule tissue [18]. The previous study also found significant levels of various growth factors and cytokines in Dupuytren's tissue, although no comparison between cord and nodule was made. In view of the differences in composition of ECM between cord and nodule that we found, and the known capacity for ECM components, such as fibronectin, to serve as a repository for growth factors [19], we expect that the profile of associated growth factors will be very different in these types of tissue. Since nodule in addition is a more "active" type of tissue, where more remodeling might be occurring, a higher release of these growth factors is also to be expected.

We found that nodule tissue shows an active profibrotic phenotype, with a high percentage of $\alpha$-SMApositive myofibroblasts, and some macrophages and high expression of fibronectin, procollagen 1 , and collagen $\mathrm{V}$. This profile bears a striking resemblance to the profile of active fibrogenic tissue as published by Blaauboer et al. [20]. In this study, the authors showed that the formation of new collagen in a mouse model for idiopathic lung fibrosis was correlated with an increased expression of type $\mathrm{V}$ collagen, elastin, tenascin $\mathrm{C}$, lysyl oxidase, and Wnt-1 inducible signaling pathway protein 1 (WISP1). In our study, we found that in nodule, there is a high expression of type $\mathrm{V}$ collagen, elastin, tenascin $\mathrm{C}$, and lysyl oxidase on mRNA and/or protein level. Furthermore, in 


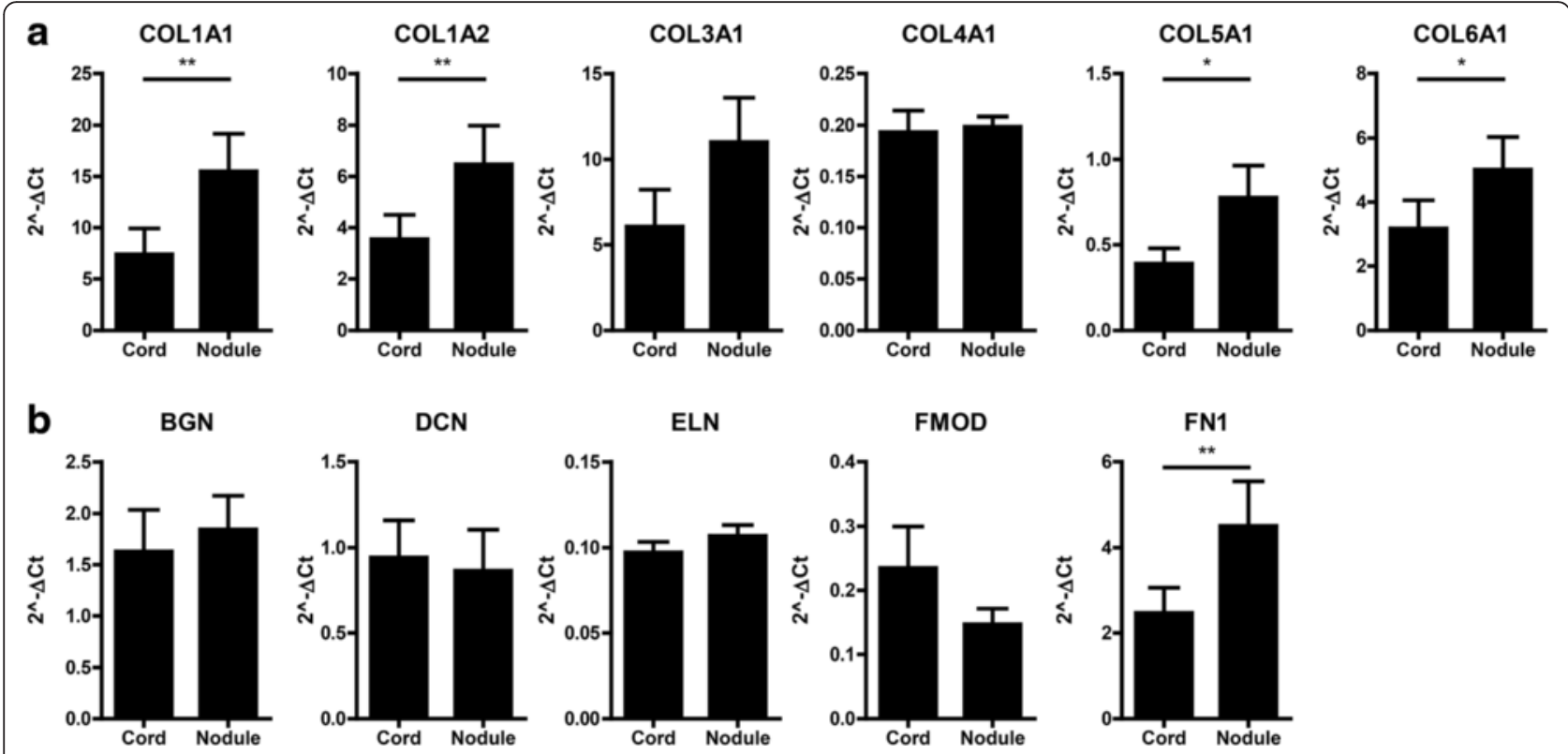

Fig. 2 Extracellular matrix mRNA profile. a Relative mRNA expression of COL1A2, COL1A2, COL3A1, COL4A1, COL5A1, and COL6A1 in cord and nodule tissue from eight Dupuytren's patients. b Relative mRNA expression of BGN, DCN, ELN, FMOD, and FN1 in cord and nodule tissue from eight Dupuytren's patients. ${ }^{*} p<0.05,{ }^{* *} p<0.01$ as determined by Wilcoxon paired rank test

a previous study, we have also found high expression of WISP1 in Dupuytren's nodules [21]. An important difference is that in our study, the expression of the fibrotic phenotype was not accompanied by the deposition of collagen I, although we found a strong increase in COL1A1 and COL1A2 mRNA and procollagen 1 protein expression.

We propose that the apparent discrepancy between collagen mRNA and deposition is caused by a difference in the expression of collagen processing molecules between cord and nodule. After the synthesis of procollagen in the fibroblast, pro-peptides are cleaved off at the $\mathrm{N}$ - and C-terminal by ADAMTSs and BMP1, respectively (reviewed in $[22,23]$ ). The cleavage of the C-terminal propeptide is essential for collagen fibril formation. Although we did not find any differences in BMP1 expression between cord and nodule at either mRNA or protein level, we did find a significantly lower expression of PCOLCE2 in nodule. BMP1 has many different functions in the cell, but only its effect on collagen processing is activated by $\operatorname{PCOLCE}(2)[24,25]$. At this moment, there is no consensus in literature about the relative contributions of the two different isoforms of PCOLCE, although they appear to have similar efficiency [26]. Intriguingly, in a study characterizing the phenotype of amniotic fluid-derived cells from fetuses with spina bifida, Hosper et al. found that these cells did not deposit any collagen, despite normal or increased levels of BMP1. These cells were then shown to have decreased levels of PCOLCE, PCOLCE2, and ADAMTSs [27]. In another study, PCOLCE2-null mice were shown to have decreased levels of collagen deposition after transverse aortic constriction [28]. Both studies give a clear indication of the essential role of these proteins in the eventual deposition of collagen by the cells.

In most studies into fibrotic conditions, the appearance of $\alpha$-SMA-positive myofibroblasts and the excessive deposition of fibrillar collagen are seen as the hallmarks of fibrosis [2]. In this study, we did not find a correlation between the expression of $\alpha$-SMA, which was higher in nodule, and the deposition of collagen, which was higher in cord. This may reflect the fact that all tissues were from patients in a later stage of Dupuytren's disease, where $\alpha$-SMA-positive myofibroblasts were originally present, but may have disappeared from the cord. Collagen, once deposited, may remain evident for a longer period than myofibroblasts, based on $\alpha$-SMA expression. Whether these myofibroblasts have reverted to normal fibroblasts or been removed by apoptosis is not known, although lower cell numbers in cord make it likely that apoptosis must play a role. For this to be true, however, it presupposes that the cells previously present in cord were capable of depositing large amounts of collagen. These therefore cannot have been equivalent to nodule myofibroblasts, which do not deposit the collagen they produce.

Apart from the differences in composition of ECM, we also found qualitative differences in the collagen itself, since significantly, more crosslinks per triple helix were present in cord tissue than in nodule. Additionally, these HP and LP crosslinks are more difficult to degrade by endogenous MMPs, as described by van der Slot-Verhoeven 


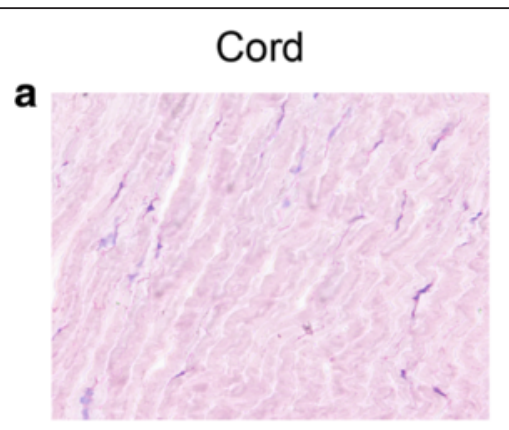

b

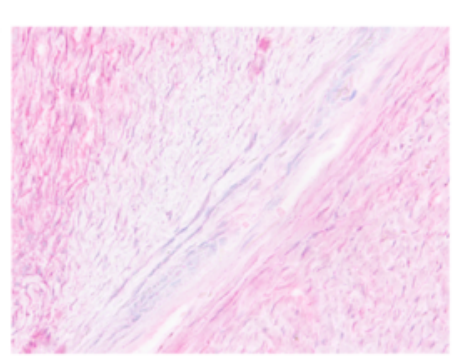

C

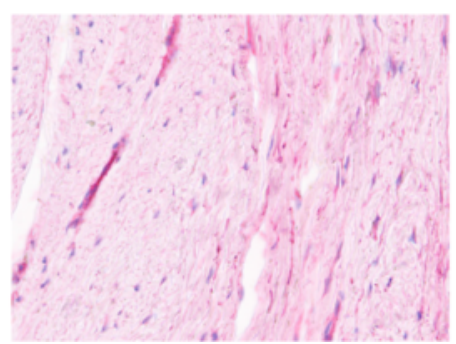

\section{Nodule}
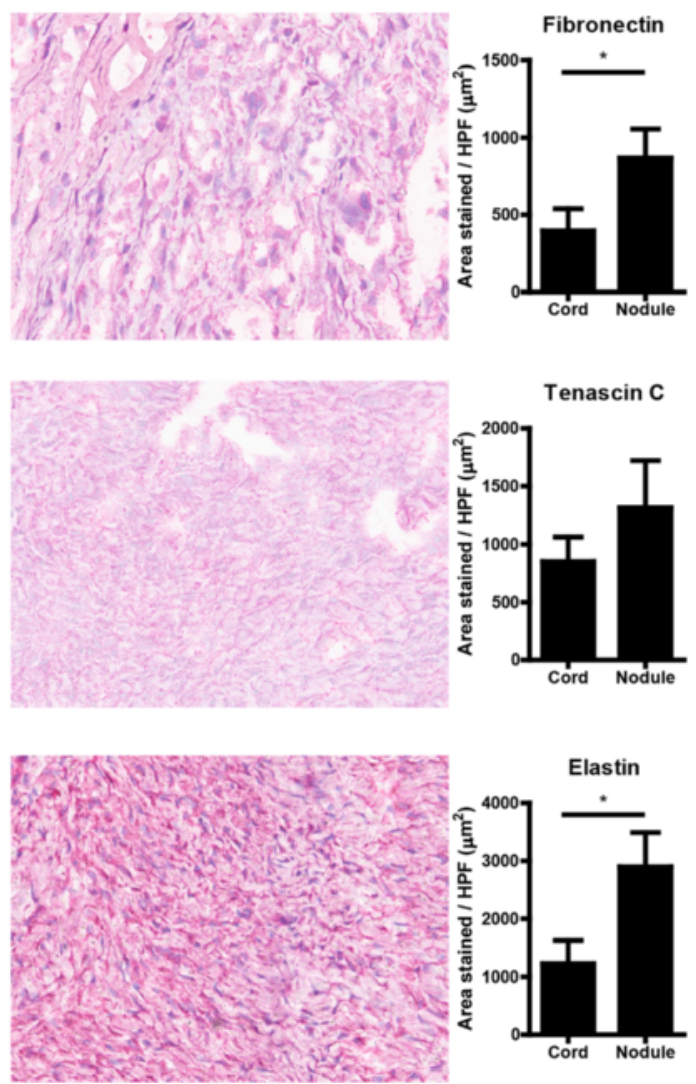

Fig. 3 Non-collagenous extracellular matrix proteins. a Representative pictures and quantification of fibronectin expression in cord and nodule tissue from ten Dupuytren's patients. $\mathbf{b}$ Representative pictures and quantification of tenascin C expression in cord and nodule tissue from ten Dupuytren's patients. c Representative pictures and quantification of elastin expression in cord and nodule tissue from ten Dupuytren's patients. ${ }^{*} p<0.05,{ }^{* *} p<0.01$ as determined by Wilcoxon paired rank test

et al. [29]. The higher crosslink levels in cord were in contradiction to a higher level of LOX mRNA in nodule, and no differences were found in the expression of LOXL1-4 and PLOD1-3. This pattern suggests that crosslinks in the cord collagen were formed previously, and in combination with the low levels of myofibroblasts and macrophages, is a profile reminiscent of previously fibrotic, now quiescent tissue, as was suggested in a previous study [17].

This may furthermore suggest that the extracellular matrix in the cord, once formed, is crosslinked and thereafter not remodeled further, with the cells responsible for the deposition of the matrix partly disappearing. The question remaining in this model is which cells are responsible for the contraction of the cord, leading to the patients' inability to extend the fingers. A previous study has noted that nodule myofibroblasts possess an inherently greater contractile ability in vitro and suggested that these cells play a large role in contraction in patients as well [5].
In other forms of fibrosis, such as liver fibrosis, it has been shown that although few fibroblasts remain after partial resolution of fibrosis, the remaining cells are more prone to reacquire a fibrogenic phenotype after a new insult [30]. This mechanism might also be present in Dupuytren's disease, where several comparative studies found that cord cells display an intermediate phenotype between nodule and normal fibroblasts $[5,6]$. One of these studies also showed that although cord cells in normal culture show less $\alpha$-SMA expression than nodule myofibroblasts, upon stimulation with TGF- $\beta 1, \alpha-S M A$ expression was upregulated to the same extent as in nodule myofibroblasts [6], suggesting that they retain fibrogenic capacity.

If nodule is indeed the more active fibrogenic tissue, and if cord remains prone to reactivation, these factors have to be taken into account in one of the current problems with the treatment of Dupuytren's disease, which is the high rate of recurrence. Recent papers suggest a slightly lower durability of the result after 

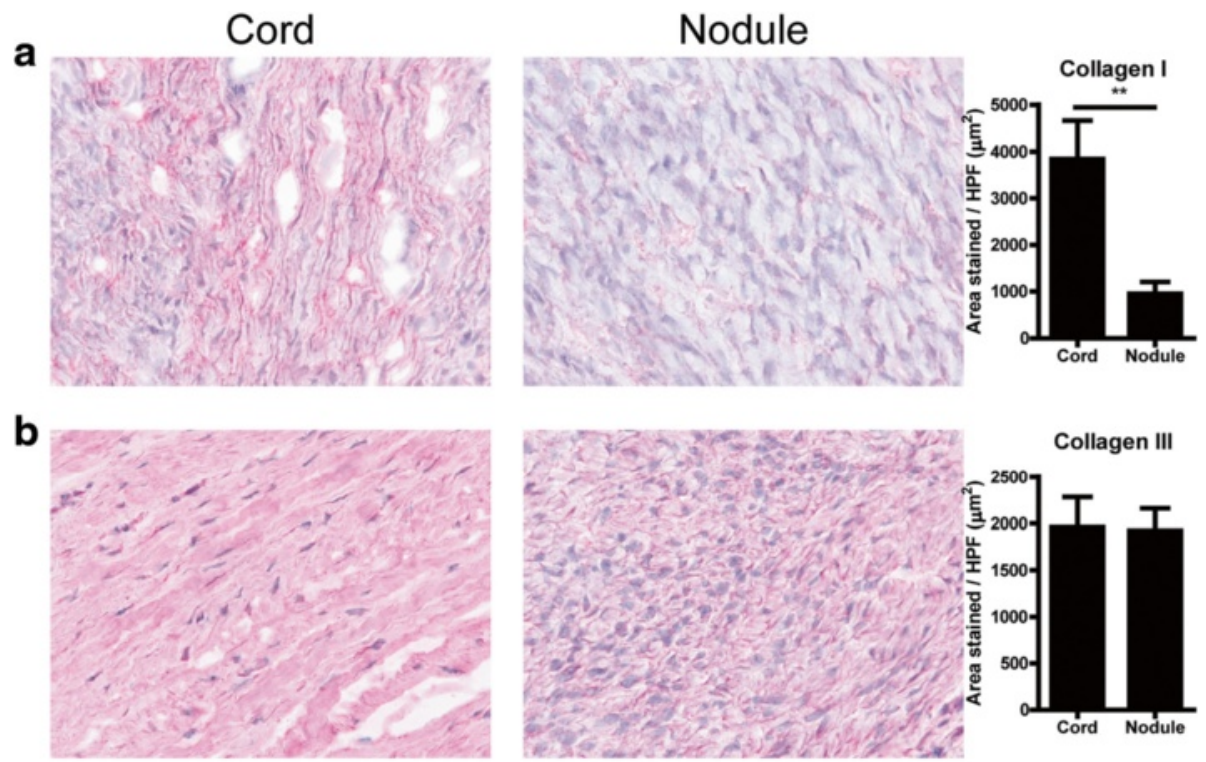

Collagen III

C
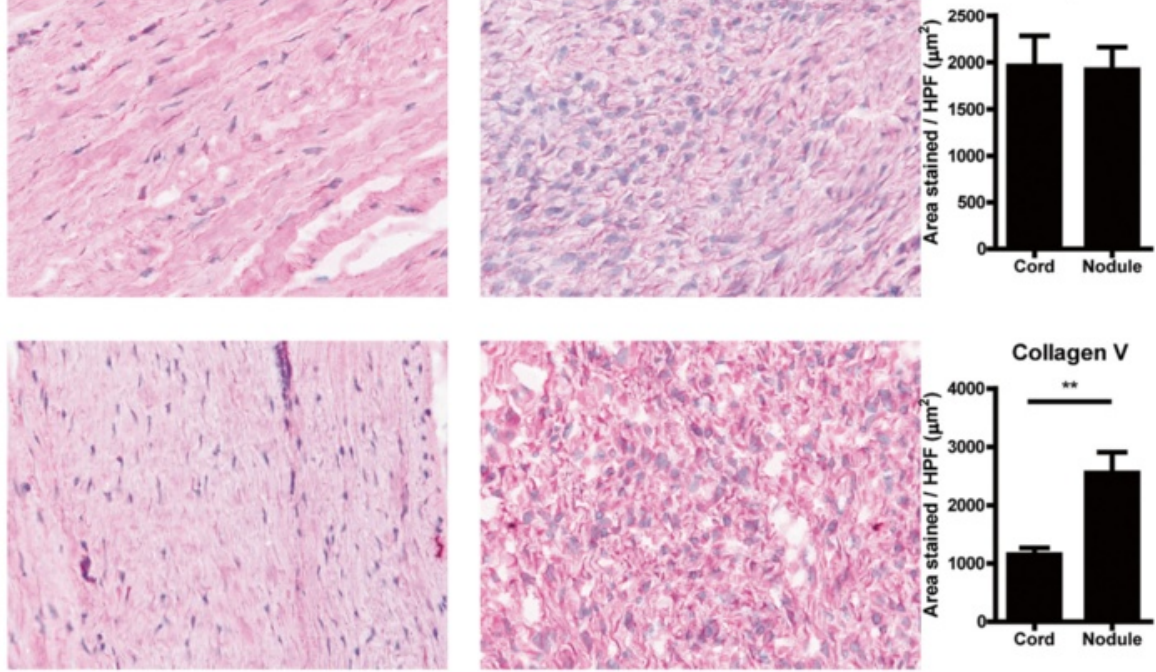

d
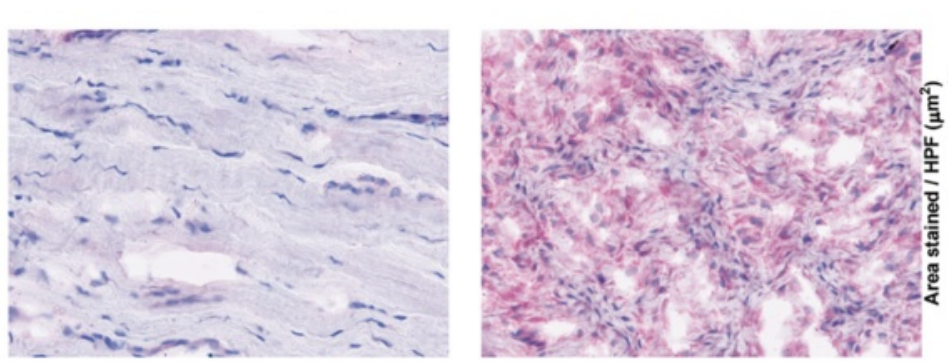

Pro-collagen 1
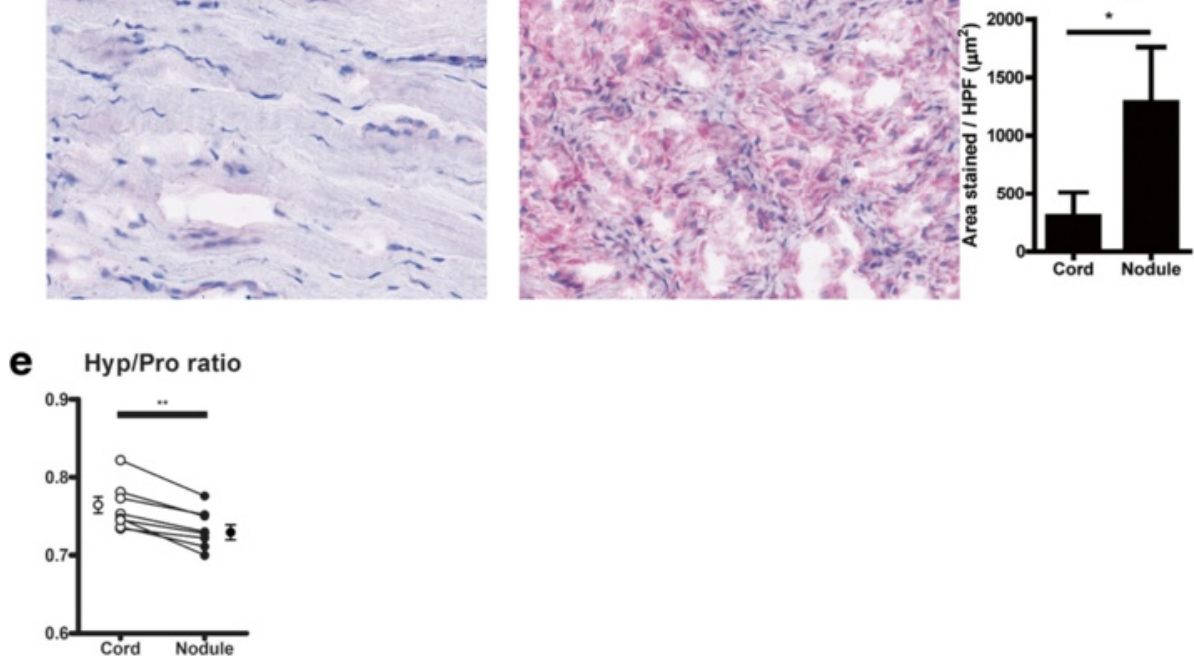

Fig. 4 Collagenous extracellular matrix proteins. a Representative pictures and quantification of collagen I expression in cord and nodule tissue from ten Dupuytren's patients. b Representative pictures and quantification of collagen III expression in cord and nodule tissue from ten Dupuytren's patients. c Representative pictures and quantification of collagen $\vee$ expression in cord and nodule tissue from ten Dupuytren's patients. $\mathbf{d}$ Representative pictures and quantification of procollagen 1 expression in cord and nodule tissue from ten Dupuytren's patients. e Graph showing the ratio between hydroxyproline and proline (Hyp/Pro) for matching cords and nodules from eight Dupuytren's patients. Dots and whiskers on both sides represent means and SEM, respectively. ${ }^{*} p<0.05,{ }^{*} p<0.01$ as determined by Wilcoxon paired rank test 


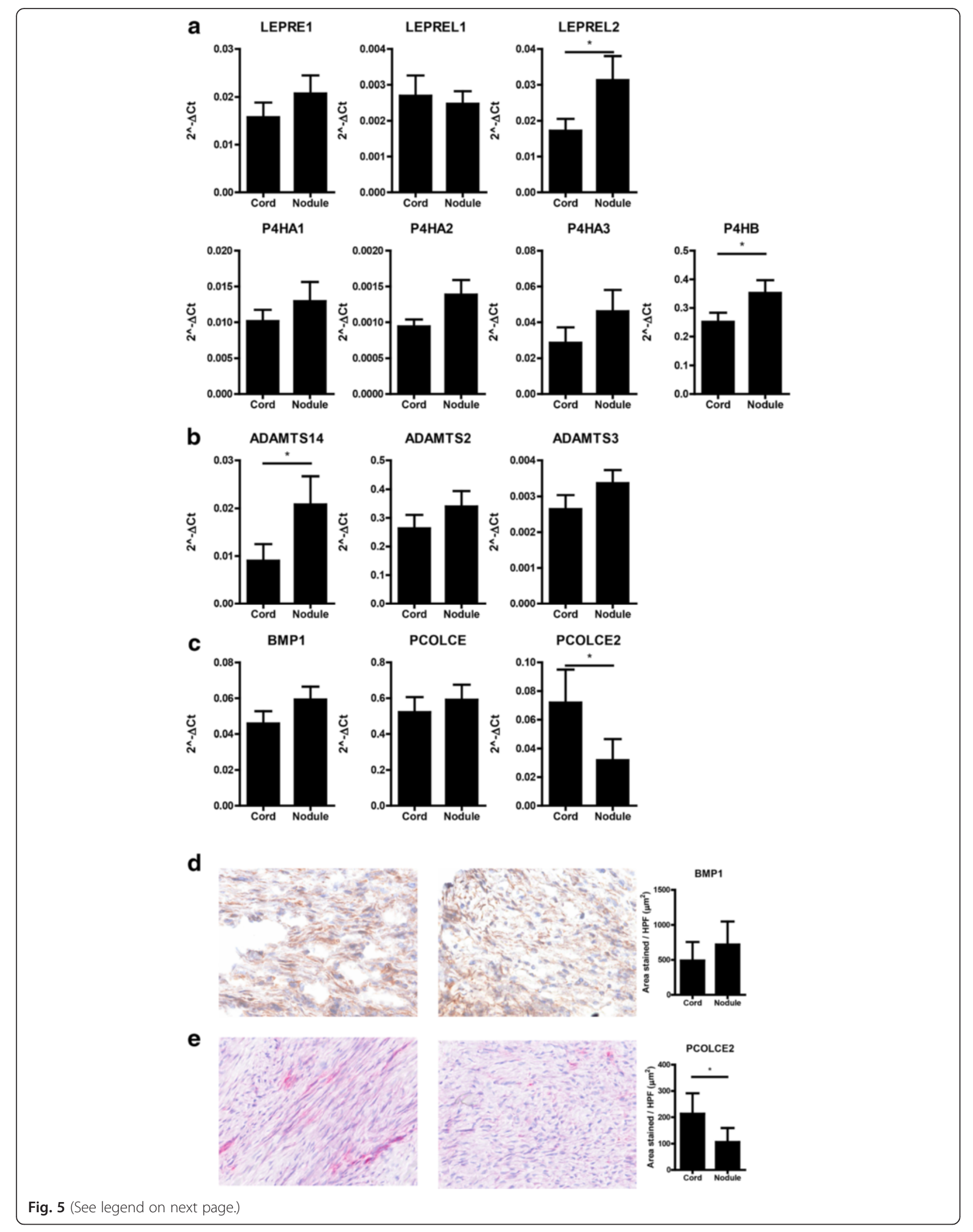


(See figure on previous page.)

Fig. 5 Collagen biosynthesis. a Relative mRNA expression of LEPRE1, LEPREL1, LEPREL2, P4HA1, P4HA2, P4HA3, and P4HB in cord and nodule tissue from eight Dupuytren's patients. b Relative mRNA expression of ADAMTS2, ADAMTS3, and ADAMTS14 in cord and nodule tissue from eight Dupuytren's patients. c Relative mRNA expression of BMP1, PCOLCE, and PCOLCE2 in cord and nodule tissue from eight Dupuytren's patients. d Representative pictures and quantification of BMP1 expression in cord and nodule tissue from ten Dupuytren's patients. e Representative pictures and quantification of PCOLCE2 expression in cord and nodule tissue from ten Dupuytren's patients. ${ }^{*} p<0.05,{ }^{* *} p<0.01$ as determined by Wilcoxon paired rank test

collagenase treatment, in which only the cord is targeted, compared to fasciectomy, in which both nodule and cord are removed [31]. Percutaneous needle fasciotomy, in which only the cord is disrupted, was also reported to have higher rates of recurrence than fasciectomy [32]. Without examination of tissue from patients with recurrent Dupuytren's disease, however, it is currently not possible to say whether reactivation of cord tissue occurs and whether this is initiated by cord fibroblasts reactivated by the procedure, by myofibroblasts from the nodule or by a different process.

\section{Conclusions}

We have found that nodule is the more pro-fibrotic tissue in Dupuytren's disease, characterized by an RNA profile consistent with fibrogenesis, but with lower levels of actual collagen I deposition, possibly caused by an abnormal collagen biosynthesis, as evidenced by a lower expression of PCOLCE2. Cord tissue contains more collagen I, which is additionally more heavily crosslinked. These differences should be taken into account when deciding the optimal treatment for Dupuytren's disease.

\section{Methods}

\section{Ethics statement}

Tissue samples were obtained following informed written consent and approval of the Medical Ethics Committee of the University Medical Centre Groningen (2007/ 067), in line with the Declaration of Helsinki.

\section{Primary tissues}

Dupuytren's nodules and cords were obtained from patients undergoing limited fasciectomy or dermofasciectomy in the University Medical Centre Groningen. Tissue from ten patients in total was used; nodules and cords of eight patients were analyzed by low density array and crosslink analysis; nodules and cords of ten patients were analyzed using immunohistochemistry.

\section{Gene expression analysis}

The expression of genes known to be involved in collagen biosynthesis and homeostasis was determined with a custom-made microfluidic card-based low-density array (Additional file 1: Table S1; Applied Biosystems, Foster City, CA). This enables accurate measurement of gene expression levels of 44 simultaneously, using a Taqman

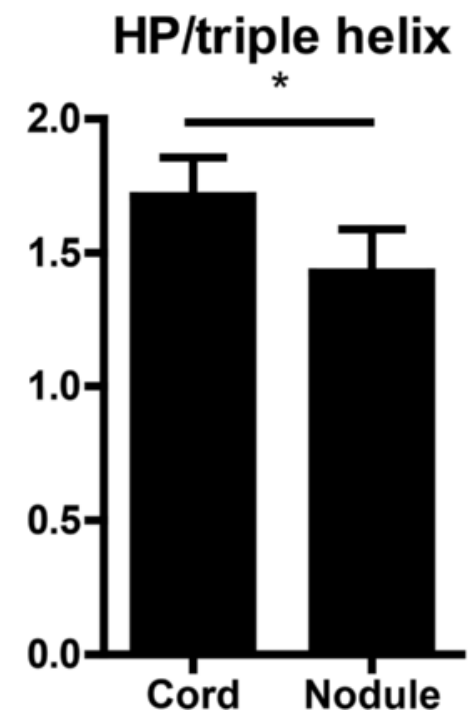

LP/triple helix

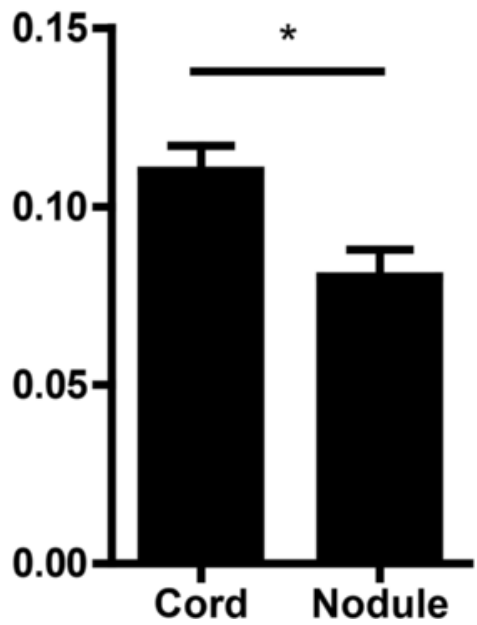

Fig. 6 Collagen crosslinks. Number of hydroxylysylpyridinoline (HP) and lysylpyridinoline (LP) residues per triple helix. ${ }^{*} p<0.05$, as determined by Wilcoxon paired rank test 
probe system. RNA was isolated from tissue using the RNeasy Fibrous Tissue Mini Kit (Qiagen), according to the manufacturer's instructions and quantified using a NanoDrop-1000 spectrophotometer (NanoDrop Technologies, Wilmington, DE). Reverse transcription was carried out using the first-strand complementary DNA (cDNA) synthesis kit (Fermentas, St. Leon-Rot, Germany). For each sample, $100 \mathrm{ng} \mathrm{cDNA}$ was diluted in $50 \mu \mathrm{L}$ of distilled water and mixed with $50 \mu \mathrm{L}$ of TaqMan PCR master mix (Applied Biosystems). Standard recommended PCR protocols were performed $\left(50^{\circ} \mathrm{C}\right.$ for $2 \mathrm{~min}, 95^{\circ} \mathrm{C}$ for $10 \mathrm{~min}$, and the next two steps were repeated for 40 cycles: $95{ }^{\circ} \mathrm{C}$ for $12 \mathrm{~s}$ and $60{ }^{\circ} \mathrm{C}$ for $1 \mathrm{~min}$ ) using the $\mathrm{ViiA}^{\mathrm{ma}} 7$ Real-Time PCR System (Applied Biosystems). Threshold cycle numbers higher than 35 were set to 35 and considered not detectable. Patients were removed from the analysis if there was no detectable expression in both cord and nodule; numbers of patients included per gene are shown in Table 1. Gene expression was calculated normalized to the geometric mean of four reference genes ( $\beta$ actin, $\beta 2$-macroglobulin, GAPDH, and YWHAZ).

\section{Immunohistochemistry}

Tissue for staining was stored at $-80{ }^{\circ} \mathrm{C}$ and cut into $5 \mu \mathrm{m}$ cryosections. The sections were air-dried for $30 \mathrm{~min}$ and fixated for $10 \mathrm{~min}$ in acetone. Washing and blocking of aspecific binding sites, endogenous peroxidases, and endogenous biotin was performed according to standard procedures. The sections were incubated for $60 \mathrm{~min}$ at RT with primary antibody (see Additional file 2: Table S2), and with species specific HRP- or biotinylated secondary antibodies (DAKO and Southern Biotech). Stainings were visualized using VECTOR Red Alkaline Phosphatase (AP) Substrate or ImmPACT DAB Peroxidase (SK5100 or SK4105, respectively, Vector Laboratories, Burlingame, CA) according to the manufacturer's instructions. All immunohistochemical stainings were counterstained with hematoxylin (Merck, Darmstadt, Germany) and mounted in Kaiser's glycerin-gelatin (Merck).

\section{Quantification of stainings}

Immunohistochemical stainings were evaluated using a Leica DM 2000 microscope. For morphometric quantification of immunohistochemistry, five representative photomicrographs at $40 \times$ magnification were taken per tissue section, using a Multispectral Imaging Camera (Perkin Elmer, Cambridge, UK). Photomicrographs were analyzed using Nuance 3.0 software (Perkin Elmer). Stained areas were quantified and expressed as square micrometer per high power field $\left(\mu \mathrm{m}^{2} / \mathrm{HPF}\right)$.

\section{Crosslink analysis}

Hydroxyproline (Hyp), proline (Pro), and the crosslinks hydroxylysylpyridinoline (HP) and lysylpyridinoline (LP) were measured in acid hydrolysates of unreduced samples by reversed-phase high-performance liquid chromatography [33, 34]. The Hyp/Pro ratio was used as a measure to estimate the ratio of collagen towards noncollagenous proteins: the lower the Hyp/Pro ratio, the more non-collagenous proteins are present in the tissue [15]. The crosslinks HP and LP are expressed as the total amount of residues per collagen molecule, assuming 300 Hyp residues per triple helix.

\section{Statistics}

Statistical analysis was performed using GraphPad Prism 5.0 using a Wilcoxon paired rank test. In all analyses, $p<0.05$ was considered to be statistically significant.

\section{Additional files}

Additional file 1: Set up of custom made microfluidic card-based low density array (Applied Biosystems, Foster City, CA). (DOCX 14 kb)

Additional file 2: Primary antibodies used. (DOCX $12 \mathrm{~kb}$ )

\section{Abbreviations}

a-SMA, a-smooth muscle actin; AP, alkaline phosphatase; ECM, extracellular matrix; HP, hydroxylysylpyridinoline; HPF, high power field; Hyp, hydroxyproline; LP, lysylpyridinoline; MMP, matrix metalloproteinases; Pro, proline; TIMP, tissue inhibitor of matrix metalloproteinase; WISP1, Wnt-1 inducible signaling pathway protein

\section{Funding}

This work was supported by a grant from the Dutch government to the Netherlands Institute for Regenerative Medicine (grant no. FES0908). The funding body did not have any influence on the design of the study and collection, analysis, and interpretation of the data and on writing of the manuscript.

Availability of data and materials

The dataset(s) supporting the conclusions of this article is (are) included within the article (and its additional file(s)).

\section{Authors' contributions}

MvB carried out all the analyses, analyzed the results, and drafted the manuscript. EtD carried out the analyses and edited the manuscript. PW participated in the design of the study, analyzed the results, and edited the manuscript. RB participated in the design of the study, analyzed the results, and edited the manuscript. All authors read and approved the final version of the manuscript.

Competing interests

The authors declare that they have no competing interests.

Ethics approval and consent to participate

Tissue samples were obtained following informed written consent and approval of the Medical Ethics Committee of the University Medical Centre Groningen (2007/067), in line with the Declaration of Helsinki.

\section{Author details}

${ }^{1}$ Department of Pathology \& Medical Biology, University Medical Center Groningen, University of Groningen, Hanzeplein 1, 9713 GZ Groningen, The Netherlands. ${ }^{2}$ Department of Plastic Surgery, Department of Pathology \& Medical Biology, University Medical Center Groningen, University of Groningen, Hanzeplein 1, 9713 GZ Groningen, The Netherlands.

Received: 28 September 2015 Accepted: 13 June 2016

Published online: 29 June 2016 


\section{References}

1. Wight TN, Potter-Perigo S. The extracellular matrix: an active or passive player in fibrosis? Am J Physiol Gastrointest Liver Physiol. 2011;301(6):G950-5.

2. Friedman SL, Sheppard D, Duffield JS, Violette S. Therapy for fibrotic diseases: nearing the starting line. Sci Transl Med. 2013;5(167):167sr1.

3. Lanting R, Broekstra DC, Werker PM, van den Heuvel ER. A systematic review and meta-analysis on the prevalence of Dupuytren disease in the general population of Western countries. Plast Reconstr Surg. 2014;133(3):593-603.

4. Rayan GM. Dupuytren disease: anatomy, pathology, presentation, and treatment. J Bone Joint Surg Am. 2007;89(1):189-98.

5. Verhoekx JS, Beckett KS, Bisson MA, McGrouther DA, Grobbelaar AO, Mudera $\checkmark$. The mechanical environment in Dupuytren's contracture determines cell contractility and associated MMP-mediated matrix remodeling. J Orthop Res. 2013;31(2):328-34.

6. Bisson MA, McGrouther DA, Mudera V, Grobbelaar AO. The different characteristics of Dupuytren's disease fibroblasts derived from either nodule or cord: expression of alpha-smooth muscle actin and the response to stimulation by TGF-beta1. J Hand Surg Br. 2003;28(4):351-6.

7. Rehman S, Xu Y, Dunn WB, Day PJ, Westerhoff HV, Goodacre R, Bayat A. Dupuytren's disease metabolite analyses reveals alterations following initial short-term fibroblast culturing. Mol Biosyst. 2012;8(9):2274-88.

8. Ulrich D, Ulrich F, Piatkowski A, Pallua N. Expression of matrix metalloproteinases and their inhibitors in cords and nodules of patients with Dupuytren's disease. Arch Orthop Trauma Surg. 2009;129(11):1453-9.

9. Johnston P, Chojnowski AJ, Davidson RK, Riley GP, Donell ST, Clark IM. A complete expression profile of matrix-degrading metalloproteinases in Dupuytren's disease. J Hand Surg [Am]. 2007;32(3):343-51.

10. Berndt A, Kosmehl H, Katenkamp D, Tauchmann V. Appearance of the myofibroblastic phenotype in Dupuytren's disease is associated with a fibronectin, laminin, collagen type IV and tenascin extracellular matrix. Pathobiology. 1994;62(2):55-8.

11. Magro G, Fraggetta F, Colombatti A, Lanzafame S. Myofibroblasts and extracellular matrix glycoproteins in palmar fibromatosis. Gen Diagn Pathol. 1997:142(3-4):185-90.

12. Magro G, Fraggetta F, Travali S, Lanzafame S. Immunohistochemical expression and distribution of alpha2beta1, alpha6beta1, alpha5beta1 integrins and their extracellular ligands, type IV collagen, laminin and fibronectin in palmar fibromatosis. Gen Diagn Pathol. 1997;143(4):203-8.

13. Luck JV. Dupuytren's contracture; a new concept of the pathogenesis correlated with surgical management. J Bone Joint Surg Am. 1959:41-A(4):635-64.

14. Vi L, Gan BS, O'Gorman DB. The potential roles of cell migration and extracellular matrix interactions in Dupuytren's disease progression and recurrence. Med Hypotheses. 2010;74(3):510-2

15. Bank RA, Beekman B, Verzijl N, de Roos JA, Sakkee AN, TeKoppele JM. Sensitive fluorimetric quantitation of pyridinium and pentosidine crosslinks in biological samples in a single high-performance liquid chromatographic run. J Chromatogr B Biomed Sci Appl. 1997;703(1-2):37-44.

16. Lam WL, Rawlins JM, Karoo RO, Naylor I, Sharpe DT. Re-visiting Luck's classification: a histological analysis of Dupuytren's disease. J Hand Surg Eur Vol. 2010;35(4):312-7.

17. Verjee LS, Midwood K, Davidson D, Essex D, Sandison A, Nanchahal J. Myofibroblast distribution in Dupuytren's cords: correlation with digital contracture. J Hand Surg Am. 2009;34(10):1785-94.

18. Verjee LS, Verhoekx JS, Chan JK, Krausgruber T, Nicolaidou V, Izadi D, Davidson D, Feldmann M, Midwood KS, Nanchahal J. Unraveling the signaling pathways promoting fibrosis in Dupuytren's disease reveals TNF as a therapeutic target. Proc Natl Acad Sci U S A. 2013;110(10):E928-37.

19. Zhu J, Clark RA. Fibronectin at select sites binds multiple growth factors and enhances their activity: expansion of the collaborative ECM-GF paradigm. J Invest Dermatol. 2014;134(4):895-901.

20. Blaauboer ME, Emson CL, Verschuren L, van Erk M, Turner SM, Everts V, Hanemaaijer R, Stoop R. Novel combination of collagen dynamics analysis and transcriptional profiling reveals fibrosis-relevant genes and pathways. Matrix Biol. 2013;32(7-8):424-31.

21. van Beuge MM, Ten Dam EJ, Werker PM, Bank RA. Wnt pathway in Dupuytren disease: connecting profibrotic signals. Transl Res. 2015;166(6):762-71.

22. Bekhouche M, Colige A. The procollagen N-proteinases ADAMTS2, 3 and 14 in pathophysiology. Matrix Biol. 2015;44-46C:46-53.

23. Vadon-Le Goff S, Hulmes DJ, Moali C. BMP-1/tolloid-like proteinases synchronize matrix assembly with growth factor activation to promote morphogenesis and tissue remodeling. Matrix Biol. 2015;44-46C:14-23.
24. Hopkins DR, Keles S, Greenspan DS. The bone morphogenetic protein 1/Tolloid-like metalloproteinases. Matrix Biol. 2007:26(7):508-23.

25. Moali C, Font B, Ruggiero F, Eichenberger D, Rousselle P, Francois V, Oldberg A, Bruckner-Tuderman L, Hulmes DJ. Substrate-specific modulation of a multisubstrate proteinase. C-terminal processing of fibrillar procollagens is the only BMP-1-dependent activity to be enhanced by PCPE-1. J Biol Chem. 2005;280(25):24188-94.

26. Steiglitz BM, Keene DR, Greenspan DS. PCOLCE2 encodes a functional procollagen C-proteinase enhancer (PCPE2) that is a collagen-binding protein differing in distribution of expression and post-translational modification from the previously described PCPE1. J Biol Chem. 2002;277(51):49820-30

27. Hosper NA, Bank RA, van den Berg PP. Human amniotic fluid-derived mesenchymal cells from fetuses with a neural tube defect do not deposit collagen type i protein after TGF-beta1 stimulation in vitro. Stem Cells Dev. 2014;23(5):555-62.

28. Baicu CF, Zhang Y, Van Laer AO, Renaud L, Zile MR, Bradshaw AD. Effects of the absence of procollagen $C$-endopeptidase enhancer-2 on myocardial collagen accumulation in chronic pressure overload. Am J Physiol Heart Circ Physiol. 2012;303(2):H234-40.

29. van der Slot-Verhoeven AJ, van Dura EA, Attema J, Blauw B, Degroot J, Huizinga TW, Zuurmond AM, Bank RA. The type of collagen cross-link determines the reversibility of experimental skin fibrosis. Biochim Biophys Acta. 2005:1740(1):60-7.

30. Kisseleva T, Cong M, Paik Y, Scholten D, Jiang C, Benner C, Iwaisako K, Moore-Morris T, Scott B, Tsukamoto H, Evans SM, Dillmann W, Glass CK, Brenner DA. Myofibroblasts revert to an inactive phenotype during regression of liver fibrosis. Proc Natl Acad Sci U S A. 2012;109(24):9448-53.

31. Muppavarapu RC, Waters MJ, Leibman MI, Belsky MR, Ruchelsman DE. Clinical outcomes following collagenase injections compared to fasciectomy in the treatment of Dupuytren's contracture. Hand (N Y). 2015;10(2):260-5.

32. van Rijssen $\mathrm{AL}$, ter Linden $\mathrm{H}$, Werker PM. Five-year results of a randomized clinical trial on treatment in Dupuytren's disease: percutaneous needle fasciotomy versus limited fasciectomy. Plast Reconstr Surg. 2012;129(2):469-77.

33. Robins SP, Duncan A, Wilson N, Evans BJ. Standardization of pyridinium crosslinks, pyridinoline and deoxypyridinoline, for use as biochemical markers of collagen degradation. Clin Chem. 1996:42(10):1621-6.

34. Bank RA, Verzijl N, Lafeber FP, Tekoppele JM. Putative role of lysyl hydroxylation and pyridinoline cross-linking during adolescence in the occurrence of osteoarthritis at old age. Osteoarthritis Cartilage. 2002;10(2):127-34.

\section{Submit your next manuscript to BioMed Central and we will help you at every step:}

- We accept pre-submission inquiries

- Our selector tool helps you to find the most relevant journal

- We provide round the clock customer support

- Convenient online submission

- Thorough peer review

- Inclusion in PubMed and all major indexing services

- Maximum visibility for your research

Submit your manuscript at www.biomedcentral.com/submit
C Biomed Central 